\title{
An Atypical Presentation of COVID-19 in a Previously Healthy Young Male With a Rare Cause of Abdominal Pain
}

\author{
Daniel R. Basharia, b, Jennifer L. Peguero-Tejada ${ }^{\text {a }}$, Jigar I. Shah ${ }^{\mathrm{a}}$
}

\begin{abstract}
Coronavirus disease 2019 (COVID-19) is an emerging infectious disease caused by a newly discovered coronavirus that has reached pandemic proportions impacting millions globally. Authors feature an atypical case of COVID-19 presenting with a rare cause of abdominal pain and severe hyperglycemia. A 22-year-old male presented to the hospital with a 1-day history of severe abdominal pain. An abdominal computed tomography (CT) scan revealed findings of epiploic appendagitis (EA) and incidentally noted suspicious pulmonary lesions suggestive of COVID-19. Radiographic findings and distinctive laboratory results led to prompt testing by nasopharyngeal polymerase chain reaction (PCR), which ultimately confirmed the proposed diagnosis. Additionally, a significantly elevated hemoglobin A1c (HbAlc) signified undiagnosed diabetes mellitus. The patient was treated conservatively with supportive measures and made a full recovery. To our knowledge, this is the first case to describe how EA, a rare cause of abdominal pain, indirectly led to the diagnosis of COVID-19.
\end{abstract}

Keywords: COVID-19; Coronavirus; SARS-CoV-2; Epiploic appendagitis; Incidental finding; Abdominal pain; Case report

\section{Introduction}

The outbreak of the novel coronavirus, severe acute respiratory syndrome coronavirus 2 (SARS-CoV-2), which causes the coronavirus disease 2019 (COVID-19) infection, was first recognized in China in December 2019 and has led to the current global pandemic. To date, there have been roughly 13 million cases worldwide with over half a million deaths due to COVID-19 [1]. Recently, there has been heightened interest

Manuscript submitted July 13, 2020, accepted July 24, 2020

Published online August 15, 2020

a'Division of Hospital Medicine, Department of Medicine, Greater Baltimore Medical Center, Baltimore, MD 21204, USA

${ }^{b}$ Corresponding Author: Daniel R. Bashari, Department of Medicine, Greater Baltimore Medical Center, 6701 N. Charles St., Baltimore, MD 21204, USA. Email: danielbasharimd@gmail.com

doi: https://doi.org/10.14740/jocmr4285 in the various clinical presentations of COVID-19. Several reports have indicated that there have been a substantial number of cases of COVID-19 presenting with atypical symptoms. We present a case of a young male presenting to our community academic hospital with a 1-day history of severe abdominal pain. COVID-19 was ultimately diagnosed after typical pulmonary findings were incidentally noted on an abdominopelvic computed tomography (CT) scan.

\section{Case Report}

A 22-year-old Hispanic male presented to our emergency department (ED) in late March 2020 for severe acute-onset abdominal pain of 1-day duration. He further described the pain as right-sided, non-radiating, and without any specific exacerbating or alleviating factors. There was no associated nausea, vomiting, diarrhea, or constipation. He endorsed subjective fevers over the preceding day. He denied any recent respiratory tract symptoms such as cough, congestion, sore throat, nasal symptoms, or shortness of breath. Detailed review of systems was also negative for any other focal infectious symptoms, such as urinary symptoms, rash, arthalgias, or myalgias. He denied any recent travel or any known sick contacts. He reported living with his girlfriend, sister, and his sister's two young children, all of which had not been sick recently. He denied tobacco or alcohol use. He reported working in construction as his occupation.

The patient had no known medical problems and surgical history only included a laparoscopic appendectomy. Initial vital signs revealed low-grade fever of $100.5^{\circ} \mathrm{F}$, tachycardia with heart rate of $119 / \mathrm{min}$, and stable blood pressure of 138/78 $\mathrm{mm} \mathrm{Hg}$ on arrival. He did not have any hypoxia or tachypnea. Body mass index (BMI) was $29.7 \mathrm{~kg} / \mathrm{m}^{2}$. Physical examination was notable for a young male who appeared non-toxic and not in any distress. The abdominal exam revealed mild tenderness upon deep palpation to the right abdomen without distention, rebound tenderness, or guarding. The lung exam was notable for clear breath sounds bilaterally.

Laboratory values (Table 1), on admission, were notable for leukopenia with neutropenia including an absolute neutrophil count (ANC) of 1,338, thrombocytopenia with platelets of $136,000 / \mu \mathrm{L}$, and hyperglycemia with glucose of $332 \mathrm{mg} / \mathrm{dL}$. Other laboratory results were as follows: elevated C-reactive 
Table 1. Laboratory Results

\begin{tabular}{|c|c|c|c|c|}
\hline Study (Unit) & Reference range & Hospital day 1 & Hospital day 2 & Hospital day 6 \\
\hline White blood cell (cells/ $\mu \mathrm{L})$ & $4,000-11,000$ & 2,530 & 2,330 & 2,980 \\
\hline Neutrophil (\%) & & 52.9 & 36.5 & 23 \\
\hline Lymphocyte (\%) & & 36 & 52.4 & 63 \\
\hline Platelets (cells/ $\mu \mathrm{L})$ & $150,000-450,000$ & 136,000 & 119,000 & 167,000 \\
\hline ALT (IU/L) & $4-40$ & 61 & & 37 \\
\hline Ferritin $(\mathrm{ng} / \mathrm{mL})$ & $30-400$ & 845.5 & & \\
\hline C-reactive protein $(\mathrm{mg} / \mathrm{dL})$ & $<0.50$ & 0.91 & & \\
\hline Lactate dehydrogenase (IU/L) & $81-216$ & 252 & & \\
\hline Lactic acid (mmol/L) & $0.5-1.9$ & 1.5 & & \\
\hline
\end{tabular}

AST: aspartate aminotransferase; ALT: alanine aminotransferase.

protein (CRP) of $0.91 \mathrm{mg} / \mathrm{dL}$, elevated lactate dehydrogenase (LDH) of $252 \mathrm{IU} / \mathrm{L}$, and elevated ferritin of $845.5 \mathrm{ng} / \mathrm{mL}$. Liver function tests revealed mild transaminitis with an elevation of aspartate aminotransferase (AST) of $38 \mathrm{IU} / \mathrm{L}$ and alanine aminotransferase (ALT) of $61 \mathrm{IU} / \mathrm{L}$. The lactic acid and procalcitonin levels were both within normal limits at $1.5 \mathrm{mmol} / \mathrm{L}$ and $<0.09 \mathrm{ng} / \mathrm{mL}$, respectively. During his workup in the ED, CT scan of the abdomen/pelvis with intravenous (IV) contrast was obtained and described inflammatory stranding centered around an ovoid fat-attenuation structure arising from the antimesenteric border of the sigmoid colon, findings consistent with acute epiploic appendagitis (EA) (Fig. 1). Secondly, the CT scan revealed ground-glass opacities (GGOs) located in the periphery of bilateral lung bases (Fig. 2), an incidental finding immediately understood to be crucial. Given the suspicious radiographic features of COVID-19 infection, he was deemed to be a person under investigation (PUI) and admitted to the hospitalist service. Droplet and contact precautions ensued along with nasopharyngeal SARS-CoV-2 testing.

On day 2 in hospital, the patient reported abdominal pain located in his left lower quadrant and denied any residual pain on the right. Considering the finding of EA on his CT scan, the gastroenterology service was consulted. The gastroenterology team recommended supportive care including a trial of nonsteroidal anti-inflammatory drugs (NSAIDs) and a low-fat diet with expected resolution of symptoms given the self-limiting nature of the condition. The patient's abdominal pain had improved by day 3 in hospital and resolved by day 4 in hospital.

Follow-up laboratory studies included a markedly elevated $\mathrm{HbA} 1 \mathrm{c}$ at $12.7 \%$, indicating a diagnosis of diabetes mellitus. The endocrinology team was consulted to assist in managing his newly diagnosed diabetes, which was treated with basal and bolus insulin therapy. An infectious disease consultation was obtained on day 4 in hospital due to intermittent fevers with neutropenia in a PUI patient. Further diagnostic testing was obtained, and supportive care measures were recommended. IV fluids were administered for hydration. Rapid influenza A and $\mathrm{B}$ antibody testing returned negative. Urinalysis returned negative for evidence of infection. Respiratory viral panel had been pending throughout his hospitalization and returned roughly one week after discharge with rhinovirus positivity. Epstein-Barr virus $(\mathrm{EBV})$ testing returned negative for the immunoglobin $\mathrm{M}$ (IgM) antibody and positive for the IgG antibody, suggestive of past infection. Human immunodeficiency virus (HIV) testing returned negative. SARS-CoV-2 ribonucleic acid (RNA) quantitative reverse transcription-polymerase chain reaction (qRT-PCR) had been pending throughout his hospital stay.

On day 5 in hospital, the patient reported mild dyspnea on exertion. His oxygen saturation remained normal throughout his entire hospitalization. A chest X-ray was ordered which described left lower lung discoid atelectasis without evidence of infiltrate. The patient's dyspnea on exertion resolved by the next morning on day 6 in hospital, and he was subsequently discharged home. He had been afebrile for approximately 28 $\mathrm{h}$ prior to discharge and his leukopenia and thrombocytopenia had improved since admission. The day after the patient had been discharged, the SARS-CoV-2 PCR returned positive. The patient was contacted and instructed to self-isolate for at least 14 days and to instruct family members at home to contact their health care providers should they develop symptoms.

\section{Discussion}

COVID-19 is an infectious disease caused by SARS-CoV-2 that was first identified in December 2019 in China. The first case documented in the US dates back to late January 2020 [2]. The COVID-19 outbreak was declared a pandemic in March 2020 by the World Health Organization [3]. Common symptoms include fever, cough, and shortness of breath or difficulty breathing. Less common symptoms include those that may be seen with other viral illnesses, including sore throat, 


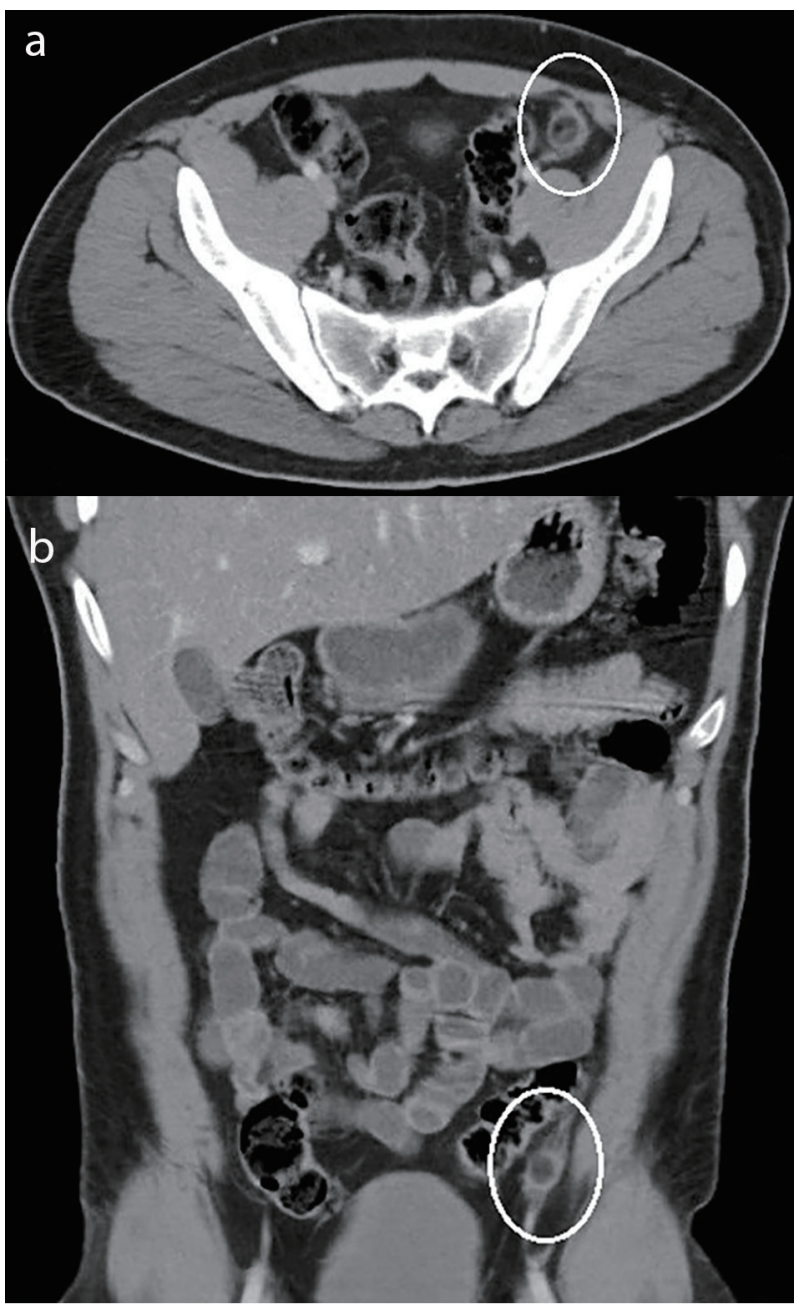

Figure 1. Contrast-enhanced CT image revealing inflammatory stranding centered around an ovoid fat-attenuation structure arising from the antimesenteric border of the sigmoid colon (circled), findings consistent with acute epiploic appendagitis in the transaxial plane (a) and in the coronal plane (b). CT: computed tomography.

myalgias, congestion, rhinorrhea, headache, fatigue, and malaise. Uncommon symptoms that have been described include nausea, vomiting, and diarrhea [4]. The prolonged incubation period (estimated to be up to 14 days with median of $5-6$ days [5]), efficient transmissibility [6], increasing concern for asymptomatic spread, and seemingly higher case fatality rate compared to other usual respiratory viruses have unfortunately made COVID-19 pervasive in society. The mainstay of diagnosis is currently made with nasopharyngeal RT-PCR testing, though this is used in conjunction with clinical suspicion, laboratory, and radiological factors that may also aide in arriving at the diagnosis. CT scanning of the chest may be valuable in patients with high clinical suspicion of COVID-19, though is currently not recommended for routine use [7], especially not in patients that do not exhibit respiratory symptoms. Currently, management is mainly focused on supportive care which may include supplemental oxygen, fluid therapy, as well as other measures directed toward affected organ systems. There are

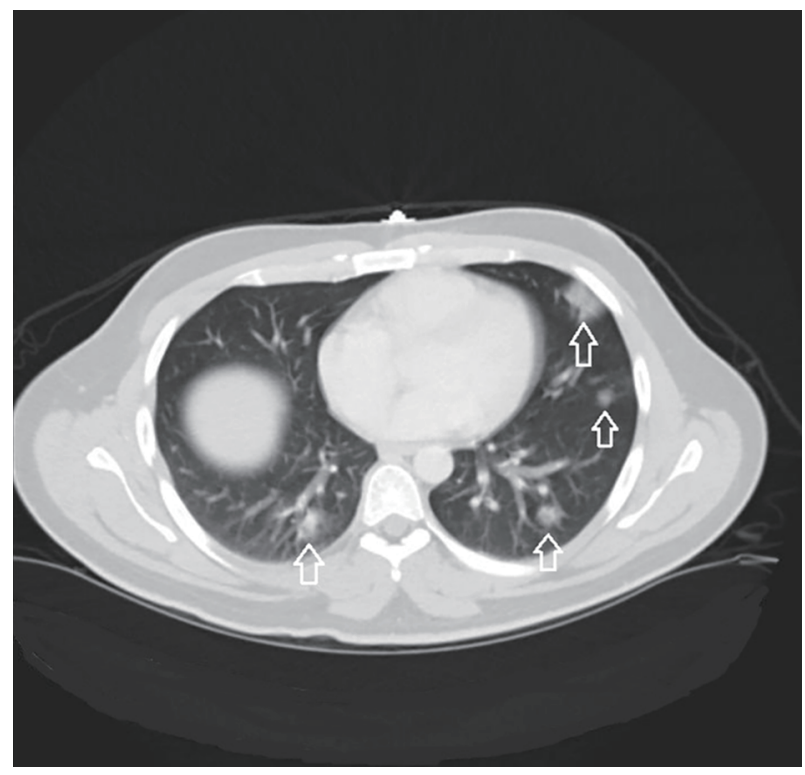

Figure 2. Image from abdominal/pelvic CT revealing patchy peripheral GGOs (arrows) present in the lung bases in the coronal plane. CT: computed tomography; GGOs: ground-glass opacities.

efforts currently underway for development of a vaccine, additional diagnostic options, as well as pharmacotherapeutics.

Recently, there has been interest in recognizing which patients with COVID-19 present with atypical symptoms who may be harboring the virus and in turn may unknowingly be transmitting it to others. Our patient was prompted to the ED primarily for abdominal pain which was not accompanied by any of the more commonly described gastrointestinal symptoms, such as diarrhea, nausea, or vomiting. In a single-center case series from Wuhan, China it was found that only up to $10 \%$ of patients presented with gastrointestinal symptoms including diarrhea and nausea [8]. It is worth mentioning that abdominal pain was the rarest of any symptom to be experienced by patients in this study [8]. Furthermore, abdominal pain as a symptom has not even elevated to a level that has been reported by other studies $[4,9]$, further affirming how rare or non-existent it may be in patients with COVID-19.

In a retrospective study [10] conducted in China, it was noted that certain hallmark findings of COVID-19 included bilateral and peripheral ground-glass and consolidative pulmonary opacities. Of the 121 patients, roughly $80 \%$ had findings of GGOs of at least one lobe with or without consolidation. The study also examined the correlation between the time of initial symptom onset and subsequent chest CT findings, and it was found that the frequency of pulmonary findings was present less often in "early" disease versus "intermediate" or "late" disease groups. This ultimately tells us that imaging with chest CT has limited utility early after symptom onset although an overwhelming majority of COVID-19 patients end up having radiographic pulmonary findings. Interestingly, in our patient, he was asymptomatic from a respiratory perspective, yet his CT scan incidentally revealed peripherally located bibasilar rounded GGOs, typical findings of COVID-19 infection. To the best of our knowledge, there hasn't been any research spe- 
cifically examining a correlation between patients without respiratory or infectious symptoms and abnormal chest CT findings. This may prove useful as we detect more patients without common symptoms which we then learn have been infected. On the other hand, this may only be possible initially under a research protocol, as currently it would be difficult to justify dedicated chest imaging without any associated focal or constitutional symptomatology.

An additional point to highlight is that the patient's pulmonary findings were noted on an abdominopelvic CT scan, without concurrent chest CT, as the patient's chief complaint was abdominal pain. CT scanning of the abdomen/pelvis typically includes the bases of the lungs and in this case revealed findings highly characteristic of COVID-19, which ultimately led to prompt formal testing and diagnosis. Furthermore, the possible utility of abdominal CT scanning in patients with COVID-19 who present atypically and who end up having pulmonary findings has not been described much in the medical literature. A recent report out of Massachusetts General Hospital [11] detailed an atypical case in which a patient presented with abdominal, testicular, and back pain as well as subjective fevers who was incidentally found to have pulmonary GGOs and consolidation along with suspected colitis on an abdominal CT scan without concurrent chest CT. In that case, the patient had been tested for COVID-19 1-day prior to his ED visit and results returned positive only after he had been discharged and unbeknownst to the treating team. Our patient presented with abdominal pain and was incidentally noted to have pulmonary findings when the CT scan was not meant to necessarily capture the lung fields. We hope our case increases awareness among clinicians who encounter patients that may present to the hospital with atypical symptoms and particularly when obtaining an abdominal CT scan for other reasons. It may prove worthwhile to lending extra attention to the lung bases to screen for incidental pulmonary findings that may suggest COVID-19, especially amid the current pandemic.

Based on the available literature to date, older adults and those with underlying medical conditions are most vulnerable for acquiring infections such as COVID-19 as well as severe disease including death, if they are infected. These chronic conditions include chronic lung and heart disease, diabetes mellitus, and immunocompromised states. In our patient, hyperglycemia was noted on admission with an elevated HbA1c of $12.7 \%$ confirming diabetes mellitus in a patient that seemingly was previous healthy. Recognizing that the $\mathrm{HbAlc}$ is a test that reflects an individual's average blood glucose level over the preceding 3 months, one can infer that the diabetes had been subclinical for at least 3 months. Clinicians should be mindful of this possibility in the age of the pandemic, and ask themselves: Could my patient be harboring a chronic condition that put them at risk for COVID-19 in the first place? In our patient, it was his subclinical diabetes that seemingly placed him at risk for COVID-19 where he ended up presenting to the hospital with overt hyperglycemia as a newly diagnosed diabetic. This observation, where a subclinical condition ends up presenting as an uncontrolled newly diagnosed disease in the setting of COVID-19 infection will likely be increasing identified.

EA is a rare and often misdiagnosed cause of abdominal pain which involves inflammation of the epiploic appendages.
Epiploic appendages have been described as small serosaladen outpouchings of fat on the external surface of the colon within the peritoneal cavity. The term "EA" was first introduced in 1956 by Dockerty et al [12]. It is a relatively rare condition with an estimated incidence of eight point eight cases per one million people per year [13], though the true incidence is unknown. EA has been diagnosed in patients initially suspected of having diverticulitis and appendicitis. An inaccurate diagnosis in this setting could lead to unnecessary antibiotic therapy and even surgical intervention. Primary EA is said to be caused by torsion of an epiploic appendage or spontaneous venous thrombosis of a draining appendageal vein [14] and secondary EA is usually associated with inflammation of adjacent organs. EA is typically diagnosed radiographically by CT scan or ultrasound. It is largely a benign and self-limiting condition with symptoms typically resolving within 3 to 14 days [15]. Supportive therapy, including NSAIDs, is the mainstay of treatment in most cases.

The pathogenesis of primary EA involves inflammation and venous thrombosis in some cases; this as we are gradually learning how COVID-19 is associated with increased hypercoagulability [16]. Recent findings have led to guidance in carefully assessing thromboembolic risk beyond routine use of risk assessment models to make mention that the existing models may not capture the true thrombotic risk COVID-19 patients carry [17]. It is unclear if the diagnosis of EA in this case is at all related to the COVID-19 infection as there have not been any previously described cases and it would be presumptuous to establish a firm association. Nonetheless, we would like readers to recognize the entity of EA in and of itself as well as the thromboembolic pathology that has been increasingly detected in patients with COVID-19.

\section{Conclusions}

Our case exemplifies an atypical presentation of COVID-19 and highlights a rare cause of abdominal pain. COVID-19 should be considered in patients with certain diagnostic cues despite presenting with unlikely symptoms, especially as we are still learning about this novel disease. It is crucial for clinicians across various disciplines to recognize the entity of EA along with its conservative management approach, to avoid unnecessary intervention. It is unclear whether EA was associated with COVID-19 in our patient, though nonetheless, it's occurrence indirectly led to the prompt diagnosis of the latter. The interrelatedness between how diabetes predisposed our patient to COVID-19, while simultaneously, COVID-19 exposing the previously subclinical diabetes is a valuable learning point in how disease states can unfold. Lastly, we remind clinicians that while incidental radiographic findings can pose diagnostic dilemmas, they sometimes can aide in arriving at an unexpected diagnosis, as was seen in our case.

\section{Acknowledgments}

None to declare. 


\section{Financial Disclosure}

None to declare.

\section{Conflict of Interest}

None to declare.

\section{Informed Consent}

The informed consent was obtained by the treating physicians.

\section{Author Contributions}

DRB contributed to conception, literature review, drafting, and revising of the manuscript. JLPT contributed to revising of the manuscript. JIS contributed to revising of the manuscript.

\section{Data Availability}

The author declares that data supporting the findings of this study are available within the article.

\section{References}

1. Johns Hopkins University. COVID-19 Map. Johns Hopkins Coronavirus Resource Center, 2020. https://coronavirus.jhu.edu/map.html. Accessed July 13, 2020.

2. Holshue ML, DeBolt C, Lindquist S, Lofy KH, Wiesman J, Bruce H, Spitters C, et al. First case of 2019 novel coronavirus in the United States. N Engl J Med. 2020;382(10):929-936.

3. WHO Director-General's opening remarks at the media briefing on COVID-19. https://www.who.int/dg/speeches/detail/who-director-general-s-opening-remarks-at-themedia-briefing-on-covid-19---11-march-2020. Published March 11, 2020. Accessed April 28, 2020.

4. Guan WJ, Ni ZY, Hu Y, Liang WH, Ou CQ, He JX, Liu L, et al. Clinical characteristics of coronavirus disease 2019 in China. N Engl J Med. 2020;382(18):1708-1720.

5. Del Rio C, Malani PN. COVID-19-new insights on a rapidly changing epidemic. JAMA. 2020;323(14):13391340.

6. Chen J. Pathogenicity and transmissibility of 2019-nCoVA quick overview and comparison with other emerging viruses. Microbes Infect. 2020;22(2):69-71.

7. Simpson S, Kay FU, Abbara S, Bhalla S, Chung JH, Chung M, Henry TS, et al. Radiological Society of North America Expert Consensus Statement on reporting chest CT findings related to COVID-19. Endorsed by the Society of Thoracic Radiology, the American College of Radiology, and RSNA - Secondary Publication. J Thorac Imaging. 2020;35(4):219-227.

8. Wang D, Hu B, Hu C, Zhu F, Liu X, Zhang J, Wang B, et al. Clinical characteristics of 138 hospitalized patients with 2019 novel coronavirus-infected pneumonia in $\mathrm{Wu}-$ han, China. JAMA. 2020;323(11):1061-1069.

9. Young BE, Ong SWX, Kalimuddin S, Low JG, Tan SY, Loh J, Ng OT, et al. Epidemiologic features and clinical course of patients infected with SARS-CoV-2 in Singapore. JAMA. 2020;323(15):1488-1494.

10. Bernheim A, Mei X, Huang M, Yang Y, Fayad ZA, Zhang $\mathrm{N}$, Diao K, et al. Chest CT findings in coronavirus disease-19 (COVID-19): relationship to duration of infection. Radiology. 2020;295(3):200463.

11. Kim J, Thomsen T, Sell N, Goldsmith AJ. Abdominal and testicular pain: An atypical presentation of COVID-19. Am J Emerg Med. 2020;38(7):1542 e1541-1542 e1543.

12. Dockerty MB, Lynn TE, Waugh JM. A clinicopathologic study of the epiploic appendages. Surg Gynecol Obstet. 1956;103(4):423-433.

13. de Brito P, Gomez MA, Besson M, Scotto B, Huten N, Alison D. Frequency and epidemiology of primary epiploic appendagitis on $\mathrm{CT}$ in adults with abdominal pain. J Radiol. 2008;89(2):235-243.

14. Pines B, Rabinovitch J, Biller SB. Primary torsion and infarction of the appendices epiploicae. Arch Surg. 1941;42(4):775-787.

15. Akinosoglou K, Kraniotis P, Thomopoulos K, Assimakopoulos SF. Epiploic appendagitis: a non-surgical cause of acute abdomen. Ann Gastroenterol. 2015;28(2):296-298.

16. Klok FA, Kruip M, van der Meer NJM, Arbous MS, Gommers D, Kant KM, Kaptein FHJ, et al. Incidence of thrombotic complications in critically ill ICU patients with COVID-19. Thromb Res. 2020;191:145-147.

17. Wang T, Chen R, Liu C, Liang W, Guan W, Tang R, Tang $\mathrm{C}$, et al. Attention should be paid to venous thromboembolism prophylaxis in the management of COVID-19. Lancet Haematol. 2020;7(5):e362-e363. 